\title{
Mini-Excitons and Lattice Dynamics in Mixed CT-Crystals: An ESR, Optical and Raman Spectroscopical Study
}

\author{
E. Erdle and H. Möhwald \\ Dornier System GmbH, NT, Friedrichshafen.
}

Z. Naturforsch. 35 a, 236-243 (1970) ; received December 18, 1979

Mixed CT-crystals of composition anthracene ${ }_{c}$ phenanthrene $e_{-c}$-tetracyanobenzene $\left(\mathrm{Anth}_{c} \mathrm{Phen}_{1-c}\right.$ TCNB, $0 \leqq c \leqq 1)$ have been investigated using ESR, optical and Raman spectroscopy. The data yield information on structural features and exciton transport as well as on the interrelation between these properties.

With respect to their structural properties the mixed crystals can be separated into two classes: a) Crystals with excess phenanthrene $(c \leqq 0.5)$ behave like Phen-TCNB exhibiting two donor orientations and lacking molecular motion as well as a phase transition. b) Crystals with excess anthracene $(c>0.5)$ are comparable to Anth-TCNB: they also undergo a first order phase transition, though at a lower transition temperature $T_{\mathrm{t}}\left(T_{\mathrm{t}}=155 \mathrm{~K}\right.$ for $c=0.75$, whereas $T_{\mathrm{t}}=206 \mathrm{~K}$ for $c=1)$.

Mini-triplet-excitons confined to the anthracene moiety were found. Their diffusion takes place quasi-onedimensionally and independently of temperature, i. e. especially without being affected by the phase transition. This result holds for the fast component of the diffusion tensor as well as for the slow one.

\section{Introduction}

The energy transport properties of crystals of weak CT complexes derive their increasing scientific interest mainly from two features:

i) The strength of exciton-phonon-coupling varies drastically for different systems, especially in the case of triplet excitons $[1,2]$.

ii) Energy diffusion is expected to be preferentially onedimensional.

On the other hand many reports continue to appear on molecular dynamics and phase transitions in these systems $[3,4]$. These are of importance as examples for phase transitions in quasi-1D-systems induced by a freezing in of large amplitude librational motions. They also serve as model systems to conclude on motional effects that are supposed to occur in organic radical ion salts [5].

These studies have up to now not dealt with the question of a connection between transport properties and phase transitions, a question that is the central one in the investigation of metal-insulator transitions in radical ion salts [6]. One system where exciton transport and a phase transition have been studied in detail is the crystal anthracene-

Reprint requests to Dr. H. Möhwald, Dornier System GmbH, NT, P.O. Box 13 60, D-7990 Friedrichshafen.
1,2,4,5 tetracyanobenzene (Anth-TCNB) [4] that was therefore chosen in this work to answer the above question.

In order to obtain additional information on the system we also investigated the structurally similar crystal phenanthrene-TCNB (Phen-TCNB) [7] and mixed crystals of the type $\mathrm{Anth}_{c} \mathrm{Phen}_{1-c}$-TCNB $(0 \leqq c \leqq 1)$ which can be prepared without any phase separation [8]. These mixed systems provide the following major advantages for our studies:

i) The influence of doping on the lattice dynamics can be studied, even at very high dopand concentrations. This then also yields information on the type of phase transition as has been successfully demonstrated for organic CT salts [9].

ii) As the excited states of Anth-TCNB are energetically more than $2000 \mathrm{~cm}^{-1}$ lower than the cor. responding ones of Phen-TCNB, high energy barriers for the diffusion of Anth-TCNB exitons are formed by the latter complexes. Thus triplet excitons in these mixed systems are delocalized over the anthracene moiety only. Furthermore they are confined in domains of neighbouring anthracenes given by a surrounding of phenanthrene molecules. The mean size of these domains can be varied by a variations of the relative concentrations of the two donors thus allowing to study excitons in a defined diffusion range.

0340-4811 / $80 / 0200-0236 \$ 01.00 / 0$. - Please order a reprint rather than making your own copy.

This work has been digitalized and published in 2013 by Verlag Zeitschrift für Naturforschung in cooperation with the Max Planck Society for the Advancement of Science under a Creative Commons Attribution-NoDerivs 3.0 Germany License. 
The experimental techniques applied in order to inspect structural as well as transport properties were triplet ESR, optical and Raman spectroscopy. Due to the very anisotropic fine structure, ESR spectroscopy is able to detect molecular orientations and their changes with high accuracy. Moreover, linewidths contain information on dynamic processes such as molecular motion or exciton diffusion. Raman experiments were performed accompanying the ESR studies, as Raman linewidths allow conclusions on the regularity of the lattice, and as the number of low frequency phonon lines may change if the system undergoes a structural phase transition. Since the delayed fluorescence exhibited by these crystals is caused by the collision of two triplet excitons its analysis yields further information on the mobility of the excitation energy.

After a description of experimental details we shall firstly present the results on the "pure" crystal Phen-TCNB. Next we shall describe ESR and Raman results on the mixed crystals, present data gained by measurements of the delayed fluorescence and finally discuss these data in the context of available information on the crystal Anth-TCNB and on organic radical ion salts, respectively.

\section{Experimental}

TCNB was a gift from the Kristallabor Stuttgart. Anthracene and phenanthrene were purified by recrystallization, vacuum sublimation and extensive zone refining.

Crystals were grown from spectroscopically pure acetone. The relative concentrations of anthracene and phenanthrene in the mixed crystals were determined as follows: After the experiments the solids were dissolved in acetone to exhibit a TCNB concentration of $10^{-4}$ mole/l. The extinction of the anthracene band at $374 \mathrm{~nm}$ (where phenanthrene and TCNB do not absorb) was compared with that of a $10^{-4}$ molar solution of anthracene. These measurements confirmed that the crystals were of the same composition (to within $\lesssim 1 \%$ ) as the solution they were grown from.

ESR measurements were performed with a Bruker $\mathrm{X}$-band spectrometer equipped with a NMR-magnetometer, a variable temperature unit using $\mathrm{N}_{2}$ gas flow or a helium continous flow cryostat (Oxford Instruments, ESR 9). Temperature measurements are estimated to be correct to about $\pm 1 \mathrm{~K}$, the temperature stabilization was better than $\pm 0.5 \mathrm{~K}$.
The crystals were irradiated by a $200 \mathrm{~W}$ mercury lamp using a $6 \mathrm{~cm}$ water filter to retain IR radiation.

Raman spectra were obtained on a Jobin-Yvon spectrometer (Ramanor HG. 2S) equipped with a photon counting assembly (photomultiplier RCA 31034). For excitation the $647.1 \mathrm{~nm}$ line of a Spectra Physics krypton ion laser (model 164-01) was used. Temperature dependent measurements were performed using an Oxford continuous flow cryostat (CF 204), whose temperature stabilization was about $\pm 0.2 \mathrm{~K}$. The accuracy of the temperature measurements is estimated to be $\pm 1 \mathrm{~K}$.

The same laser (476.2 nm line) and the same cryostat were used for measurements of the (delayed) fluorescence. For its analysis a $25 \mathrm{~cm}$-monochromator (Schoeffel) and a photomultiplier (RCA C $7151 \mathrm{~W}$ ) were applied. Decay curves were measured by a Tektronix storage-oscillograph. The chopper assembly used allowed to detect delayed fluorescence if triplet lifetimes were longer than about $0.2 \mathrm{msec}$. The estimated accuracy of the determined decay times is about $0.5 \mathrm{msec}$.

At room temperature the Phen-TCNB crystal is monoclinic $(a=9.41 \AA, b=13.1 \AA, c=7.26 \AA, \beta$ $=93.06^{\circ}$ ) with space group C2/m [7]. Phenanthrene and TCNB are stacked alternately along the $c$ axis the mean distance between donor and acceptor planes being $3.45 \AA$. The molecular planes form an angle of about $68^{\circ}$ with the stack axis. Thus this structure is extremely similar to that of Anth-TCNB [10]. Furthermore it is notable that the phenanthrene molecules with equal probability occupy two different sites which are related by a reflection through the $a c$-plane.

The mixed systems Anth $_{c}$ Phen $_{1-c}$-TCNB crystallize homogeneously as solid soulutions and due to the similarity of the structures of Anth-TCNB and PhenTCNB the two donors are expected to substitute each other most easily.

\section{Results}

\subsection{Phen-TCNB}

To study the molecular orientation in PhenTCNB we embedded triplet traps which we investigated by ESR spectroscopy. In order to study crystals of composition $\mathrm{Anth}_{c} \mathrm{Phen}_{1-c^{-}}$TCNB we used anthracene as a trap in a concentration of $c \cong 10^{-3}$. This trap was already successfully used to study the crystal naphthalene-TCNB [11]. 
At room temperature one finds two pairs of ESR lines which show the hyperfine structure (HFS) due to anthracene. At low temperatures these lines can also be detected besides some trap signals which were not investigated in this work. Increase of temperature up to $400 \mathrm{~K}$ let these anthracene signals unaffected. This leads to the conclusion that the anthracene guest molecules occupy two inequivalent sites in the Phen-TCNB host crystal for all temperatures, as do the host donors. Rotating the magnetic field in different principal molecular planes yields the following characterization of the molecular orientation:

i) The sites are mirror symmetrical with respect to the ac-plane as are the sites of the host donors. ii) A rotation of about $50^{\circ}$ about the $c$ axis and one of about $24^{\circ}$ about the long anthracene axis $x$ transforms a guest donor from one site to the other. No tilting of the molecular planes about the short axis $y$ can be detected.

No hint for any motion of the guest molecules in the temperature range between $10 \mathrm{~K}$ and $400 \mathrm{~K}$ could be found. This result was claimed by Wright et al. for the host donors [7] and is obviously corroborated by our experiments. Conclusively no structural phase transition could be observed for the crystal Phen-TCNB, as in similar system it is the onset of a librational motion between two such sites that causes the phase transition. Instead the structure

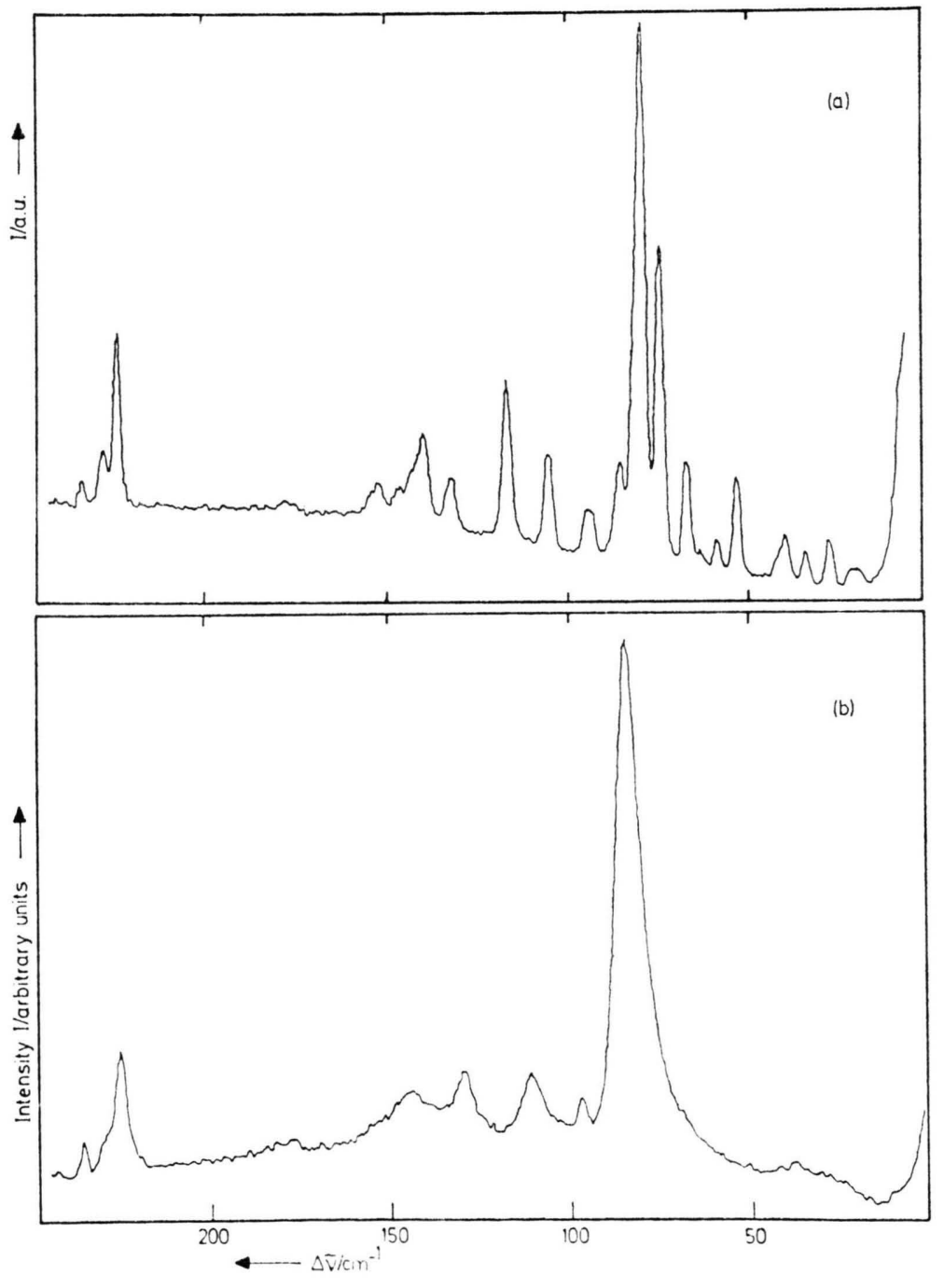

Fig. 1. Low frequency Raman spectra of Phen-TCNB (a) and Anth $_{0.5}$ Phen $_{0.5}$. TCNB (b). Excitation wavelenght: $647.1 \mathrm{~nm}$. Resolution: $2 \mathrm{~cm}^{-1}$ and $4 \mathrm{~cm}^{-1}$, respectively. 
of Phen-TCNB seems to correspond to that of AnthTCNB at low temperatures [12].

The non-existence of a phase transition is also confirmed by the Raman experiments. Figure $1 \mathrm{a}$ shows a Raman spectrum of Phen-TCNB at a temperature of $10 \mathrm{~K}$. One observes rather narrow lines (width $<2 \mathrm{~cm}^{-1}$ ) proving that the two possible sites are occupied by the phenanthrene molecules in a regular and not in a statistical manner. Thus the structure is ordered in contrast to what is supposed in [7]. Raise of temperature only results in small frequency shifts and line broadening due to lattice expansion and lifetime shortening, respectively.

\subsection{Anth Phen $_{1-c}$-TCNB crystals}

\subsection{1. $c=0.25$ and $c=0.5$}

Like in the crystal with $c \cong 10^{-3}$, Anth $_{0.25} \mathrm{Phen}_{0.75}$. TCNB and Anth $_{0.5}$ Phen $_{0.5}$-TCNB exhibit two inequivalent sites of the donors, which are again mirror symmetrical with respect to the ac-plane. However, the $x$ axes are related by a rotation about the $c$ axis of about $70^{\circ}$. This result also holds for all temperatures between $10 \mathrm{~K}$ and $300 \mathrm{~K}$. No phase transition is observed in the ESR as well as in the Raman spectra. The ESR-linewidth, a further aspect of interest, will be dealt with in section 3.2.3.

Only a few Raman modes can be observed for these mixed systems (Figure $1 \mathrm{~b}$ ). Even at $10 \mathrm{~K}$ these modes are rather broad (linewidth $>3 \mathrm{~cm}^{-1}$ ), as one expects in the case of a solid solution.

\subsubsection{Anth $_{0.75} \mathrm{Phen}_{0.25}$-TCNB}

As will be seen, Anth ${ }_{0.75} \mathrm{Phen}_{0.25}$.TCNB is the most interesting system of the mixed crystals we investigated. At room temperature one observes only one pair of ESR lines which are clearly narrower than the lines of the systems in the foregoing sections (cf. 3.2.3.) ${ }^{\S \S}$. Cooling the crystal yields a splitting of each ESR line into two signals at a temperature of about $155 \mathrm{~K}$, quite analogously to the behaviour of pure Anth-TCNB [4]. This is demonstrated in Fig. 2 where the line splittings of the pure and the mixed crystal can be compared. In both systems elevating the temperature starting from $10 \mathrm{~K}$ first leads to an only gradual decrease of the line splitting followed by a sudden collapse of that splitting. The temperature corresponding to this abrupt change is

$\$$ The crystals also exhibit optical spin polarization which was not investigated in this work.

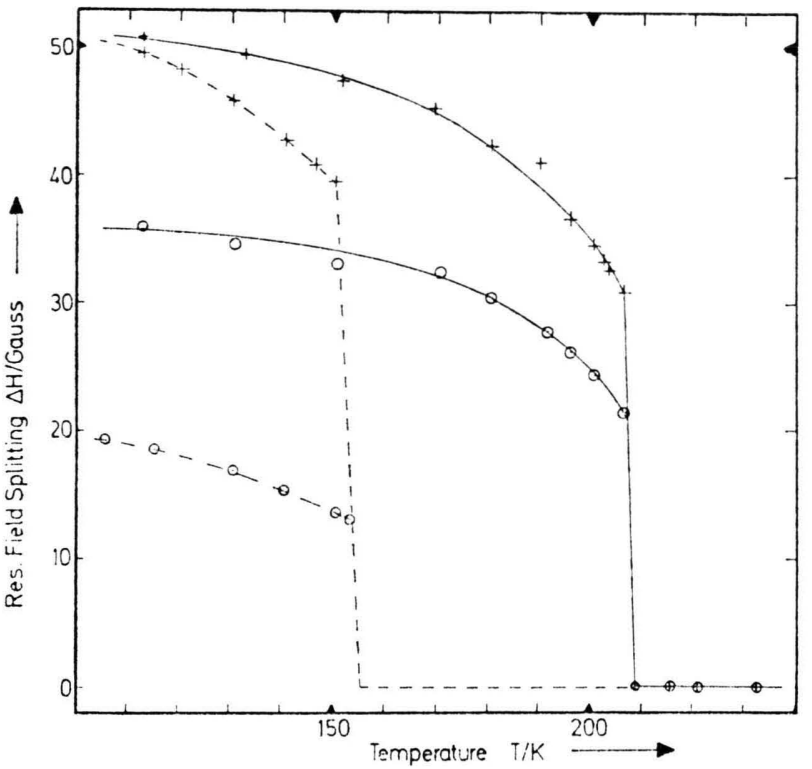

Fig. 2. ESR-resonance field splitting $\Delta H$ as a function of temperature of Anth-TCNB and Anth $_{0.75}$ Phen $_{0.25}$-TCNB (dashed curve).

apparently $50 \mathrm{~K}$ lower in the mixed than in the pure crystal.

Obviously the mixed crystal also undergoes a structural phase transition from a high temperature phase of magnetically equivalent sites to a low temperature phase of inequivalent ones. In the low temperature phase the $x$ axes of the anthracene molecules form an angle of $\pm 8.2^{\circ}$ with $x_{0}$, the mean orientation of the long molecular axes at high temperatures, as can be derived from Figure 3. There the dependence of the resonance field on the orientation is shown for temperatures above and below the transition temperature. The extrema correspond to field orientations parallel to the long donor axes $x$. Whereas in the high temperature phase there appears only one maximum at the orientation $\Phi=0^{\circ}$, in the low temperature phase $(T=102 \mathrm{~K})$ two extrema occur at $\Phi= \pm 8.2^{\circ}$. From this and from measurements of the resonance fields when rotating the magnetic field in other crystallographic planes one deduces that the long donor axes $x$ are tilted by $\pm 8.2^{\circ}$ with respect to their mean orientation in the high temperature phase. At all temperatures the two molecular sites are symmetrical with respect to a reflection through the ac-plane as they are also in the case of $c \leqq 0.5$. 


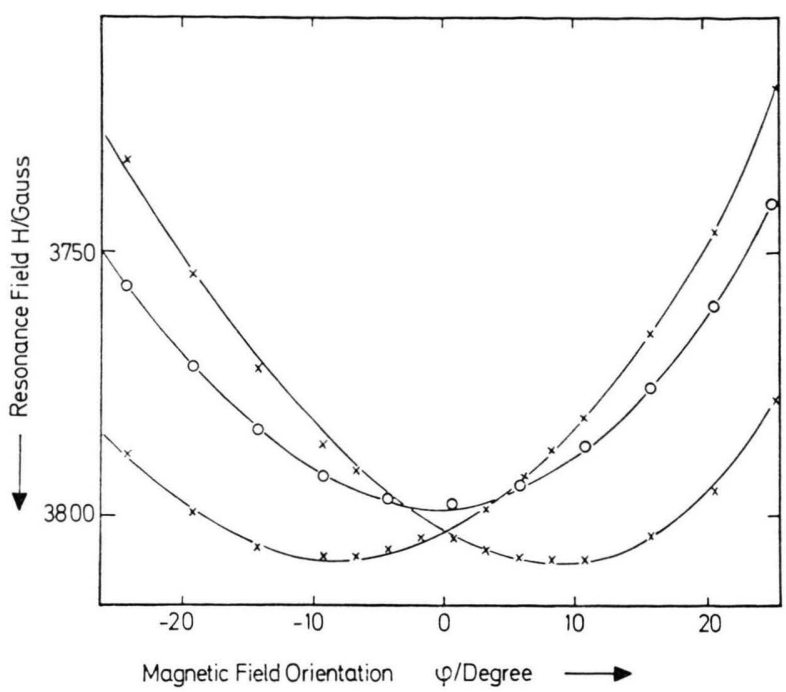

Fig. 3. Fine structure of $\mathrm{Anth}_{0.75} \mathrm{Phen}_{\mathbf{0 . 2 5}}$-TCNB. Only a small section near $H \| x(\hat{=} \varphi=0)$ is shown. The magnetic field is rotated in the molecular $x y$-plane. " $\mathrm{x} ": T=102 \mathrm{~K}$, "०": $T=295 \mathrm{~K}$. Microwave frequency: $9336 \pm 2 \mathrm{MHz}$.

Despite the lower transition temperature the similarity of the temperature dependent behaviour of Anth $_{0.75}$ Phen $_{0.25}$-TCNB and "pure" Anth-TCNB is a complete one. Like there we here observe triplet excitons wherefrom we gain information on the mean orientation of many molecules. Our measurements clearly show that this system, although being doped with guest molecules to an amount as high as $25 \%$, also undergoes a first order phase transition as a consequence of the freezing in of a donor libration about the molecular $z$ axes.

This analogous behaviour is observable in the Raman spectra, too. A new mode appears at the transition temperature, and its linewidth exhibits a similar dependence on temperature as the corresponding mode of Anth-TCNB [13]. This is demonstrated in Fig. 4 that compares the temperature dependence of frequencies and linewidths of two low frequency Raman modes which for the pure crystal are most sensitive with respect to the phase transition.

\subsubsection{Mini-Ex citons}

Table 1 presents the ESR linewidth as a function of the anthracene concentration $c$ for the two canonical orientations $H \| x$ and $H \| y$. No values for $c=0.5$ are given because in this case the linewidth is determined by statical disorder. This results in a line broadening since the inhomogeneity of molec-

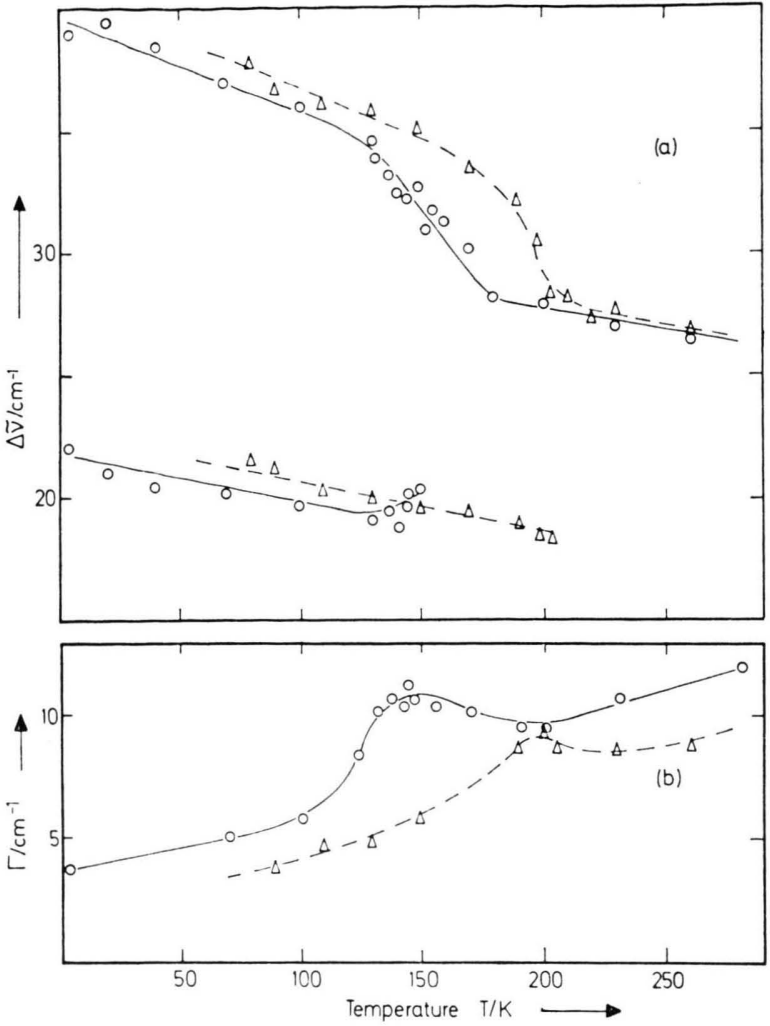

Fig. 4. Linewidths (b) and frequencies (a) of low frequency Raman modes of Anth-TCNB (dashed curves) and Anth $_{0.75} \mathrm{Phen}_{0.25}$ TCNB as functions of temperature.

ular orientations causes a distribution of resonance fields [14]. For the other systems investigated one can rule out that statical disorder determines the linewidths for $H \| \mid x$ and $H|| y$, respectively, for the following reasons:

1. For $c \cong 10^{-3}$ the HFS of the anthracene molecules in the Phen-TCNB host crystal could be resolved. Conclusively, the anthracene molecules are homogeneously aligned.

2. No orientational dependence of the linewidth has been observed in the case of Anth-TCNB [4], i. e.

Table 1. ESR-linewidths for canonical orientations as functions of the anthracene concentration $c$.

\begin{tabular}{lll}
\hline$c$ & $H \| y$ & $H \| x$ \\
\hline 0.001 & $\cong 10 \mathrm{G}$ & $\cong 16 \mathrm{G}$ \\
0.25 & $\cong 6.7 \mathrm{G}$ & $\cong 9 \mathrm{G}$ \\
0.75 & $\cong 2.3 \mathrm{G}$ & $\cong 2 \mathrm{G}$ \\
1 & $0.5 \mathrm{G}$ & $0.5 \mathrm{G}$ \\
\hline
\end{tabular}


$c=1$. This would not be expected if statical dis. order would influence the linewidth [14]. Thus it is a residual one due to excitons averaging out the HFS.

3. From the orientational dependence of the linewidth of Anth $_{0.75} \mathrm{Phen}_{0.25}$-TCNB one derives a mean misalignment of the molecular $x$ axes of less than $1^{\circ}$ which has no effect at the canonical orientations [15].

4. Finally, for $\mathrm{Anth}_{0.25} \mathrm{Phen}_{0.75}$ TCNB one gets a misalignment of the $x$ axes of less than $7^{\circ}$, that would effect linewidths of less than 3 Gauss at the canonical orientations. The measured values are more than twice as large, however, as can be seen in Table 1.

Thus the assumption is correct that statical disorder has minor or no influence at all on the linewidth at the canonical orientations. In order to obtain an explanation for the dependence of the linewidth on the anthracene concentration $c$ we have to take into consideration that we are dealing with solid solutions. Assuming that anthracene and phenanthrene are embedded statistically in the crystals, one expects the anthracene molecules to form domains which are surrounded by a boundary of phenanthrene molecules and vice versa. In view of the different electronic properties of the complexes Anth-TCNB and Phen-TCNB we further assume the excitation energy to be delocalized on the anthracene molecules only. The size of their domains depends on the anthracene concentration $c$, and the miniexcitions hopping only within these domains cause decreasing linewidths with increasing $c$ [16]. A detailed analysis based on this model, for different dimensionalities of exciton diffusion, proved its correctness and yielded the interesting result that these mini-excitons move preferentially along molecular chains in a quasi-onedimensional way [17].

\subsubsection{Delayed fluorescence}

It is well established that in Anth-TCNB the delayed fluorescence does not result from a monomolecular process (which usually is thermally activated) but from triplet-triplet-(T-T-) annihilation instead. T-T-annihilation, however, is very unlikely to occur in the case of pure onedimensional exciton diffusion, especially if along a chain high energy barriers due to phenanthrene molecules are intercalated. From measurements of the delayed fluores- cence we therefore gain information on the slow component of exciton migration perpendicular to the direction of fast hopping, which certainly takes place within the measured triplet lifetimes of about 2 msec. Figure 5 shows the intensities of the delayed and prompt fluorescence of $\mathrm{Anth}_{0.75} \mathrm{Phen}_{0.25}$-TCNB as a function of temperature, normalized to the intensities at $T=100 \mathrm{~K}$. Prompt and delayed fluorescence exhibit decreasing intensities with increasing temperature without showing any drastic change at the transition temperature of $155 \mathrm{~K}$. Denoting by $k_{T 1 S 1}$ the rate constant for T-T-annihilation to form an excited singlet state (and thus delayed fluorescence) we are led to the following relation:

$$
k_{T 1 S 1} \sim I_{\mathrm{DF}}\left(I_{\mathrm{F}}^{2} \tau_{1}^{2}\right)^{-1}
$$

where $I_{\mathrm{DF}}$ and $I_{\mathrm{F}}$ are the intensities of the delayed and prompt fluorescence, respectively, and $\tau_{1}$ is the lifetime of the triplet state. $\tau_{1}$ turns out to be determined not by T-T-annihilation but by radiative and non-radiative processes from the lowest excited triplet state to the singlet ground state alone.

In the simplest case the transition rate $k_{T 1 S 1}$ itself is linearly related to the diffusion coefficient D of the excitons [18]. Since $k_{T 1 S 1}$ does not vary with temperature, we conclude that this also holds for the slow component of exciton diffusion, i. e. especially, that it is not thermally activated. Moreover we learn that the diffusion is not affected by the phase transition, a result, that is also valid for the fast component of exciton diffusion along molecular chains $[4,15]$.

Table 2 presents the lifetimes $\tau_{1}$ as a function of the anthracene concentration $c$. Since the lifetime may be determined by the time needed until the excitation energy gets quenched at impurities or at

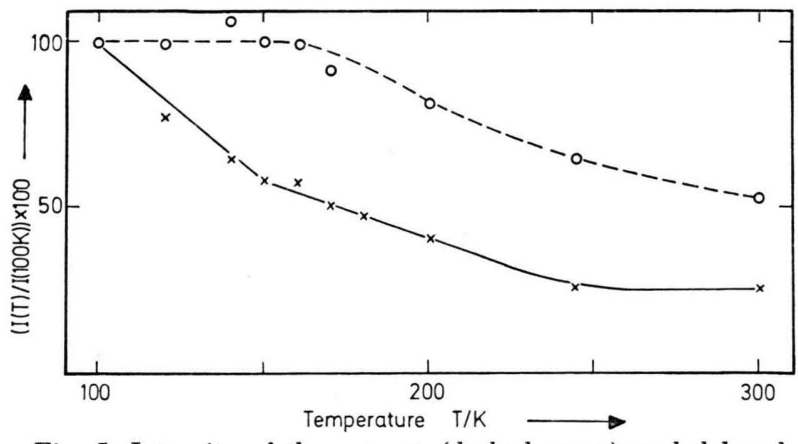

Fig. 5. Intensity of the prompt (dashed curve) and delayed fluorescence of $\mathrm{Anth}_{0.75} \mathrm{Phen}_{0.25}$-TCNB as functions of temperature. 
Table 2. Decay time (msec) of the delayed fluorescence signals as a function of temperature and anthracene concen. tration $c$.

\begin{tabular}{llll}
\hline$c / T$ & $100 \mathrm{~K}$ & $150 \mathrm{~K}$ & $290 \mathrm{~K}$ \\
\hline 0.001 & 6 & 6 & - \\
0.25 & 3.7 & 3.7 & 4 \\
0.5 & 3.4 & 3.4 & 3 \\
0.75 & 2.2 & 1.7 & 2 \\
\hline
\end{tabular}

lattice imperfections, the increase of the decay times with decreasing $c$ is reasonable. The time until quenching occurs will certainly be longer if there are less anthracene molecules for the excitons to migrate on.

\section{Discussion}

The data presented above shall yield information on two charge transfer crystals which in addition can also be grown as mixed solid solutions. The results present details on the structure, on the energy transfer and on the interrelationship between these features in these systems, which shall be briefly discussed.

\subsection{Structural aspects}

Raman as well as ESR data proved that no phase transition of the crystal Phen-TCNB exists between $10 \mathrm{~K}$ and $400 \mathrm{~K}$. This is conceivable as it was also shown that in this temperature range no donor motion takes place and as the onset of donor librations is the reason for the occurrence of a phase transition. The non-existence of a donor libration in Phen-TCNB is also of interest in view of the fact that phenanthrene, as a guest in the crystal naphthalene-TCNB, does not librate, whereas other donors like naphthalene, anthracene or pyrene do [20]. Thus it may be the unique sickle-like shape of phenanthrene or its special interaction with TCNB which prevent it from motion.

Whereas the just mentioned results agree with the $\mathrm{X}$-ray analysis, a discrepancy exists with respect to the order of the crystal structure. Our low temperature Raman data clearly indicate the crystal PhenTCNB to be ordered. The fact that only two distinct anthracene (guest) orientations are observed shows that no more than two differently oriented donor sites exist.
The mixed crystals of composition Anth $_{c} \mathrm{Phen}_{1-c^{*}}$. TCNB can be separated in two different classes with respect to their structure:

1. $c \leqq 0.5$ : crystals with excess phenanthrene behave like Phen-TCNB, exhibiting two different donor sites and neither a phase transition nor molecular motion.

2. $c>0.5$ : crystals with excess anthracene are structurally similar to Anth-TCNB. A first order phase transition is observed as a consequence of the freezing in of an in plane anthracene motion, with considerably reduced transition temperature $T_{t}$, however. In going from $c=1$ to $c=0.75 T_{t}$ is reduced from $206 \mathrm{~K}$ to $155 \mathrm{~K}$. Analogous to the argumentation with doping effects in radical ion salts [9] this drastic reduction on donor doping is understood as it are the crystallographic (ab-) planes containing the donors which are mainly affected by the phase transition [12].

\subsection{Triplet excitons}

One remarkable feature of the mixed systems results from the fact that the excited states of AnthTCNB are considerably lower in energy than the corresponding ones of Phen-TCNB. Thus AnthTCNB excitons can be studied which are confined in cages formed by phenanthrene molecules surrounding the anthracene domains. The mean size of the domains can be varied via the relative donor concentrations. To a certain extent the results on these so-called mini-excitons will then also be representative of the perfect crystals.

The fact that up to an anthracene concentration $c$ of 0.5 and up to a temperature of $400 \mathrm{~K}$ two and only two distinct ESR lines are observable shows that the exciton hopping frequency between different sites is at least smaller than $10^{7} \mathrm{sec}^{-1}$. Otherwise the two lines would be either exchange broadened or a third line would appear at a field nearly in the middle between the other ones. Although not being an exact proof this is an argument against an interpretation of Vyas and Goncalves [21], who assume a thermally activated triplet exciton transfer between differently oriented anthracene molecules in AnthTCNB.

A model on the concentration dependence of the ESR linewidth demonstrates that exciton transfer 
occurs predominantly into one direction [17]. How. ever, at present there is no experiment available to find out if this direction is along the stack axis $c$ or perpendicular to it.

Delayed fluorescence of Anth-TCNB is observed for anthracene concentrations even as low as $25 \%$. As in these mixed systems it is hardly possible for two excitons to collide by solely moving within chains which are frequently interrupted, these experiments implicitly measure the slow component of exciton diffusion perpendicular to the direction of the fast transport. As the rate constant $k_{T_{1} S_{1}}$ for $\mathrm{T}$. $T$-annihilation decreases with increasing temperature one may conclude that also this type of triplet diffusion is not thermally activated. This was previously also observed for the large component of the diffusion tensor and is conceivable, as for the unpolar triplet excitons in Anth-TCNB only a weak exciton phonon coupling is expected.

[1] H. Möhwald and E. Sackmann, Chem. Phys. Lett. 21, 43 (1973) ; H. Möhwald and E. Sackmann, Z. Naturforsch. 29 a, 1216 (1974).

[2] D. Haarer, Chem. Phys. Lett. 27, 91 (1974) ; 31, 192 (1974).

[3] C. A. Fyfe, J. Chem. Soc. Faraday II 70, 1633, 1642 (1974) ; C. A. Fyfe, D. Harold-Smith, and J. Ripmeester, J. Chem. Soc. Faraday II 72, 2269 (1976).

[4] H. Möhwald, E. Erdle, and A. Thaer, Chem. Phys. 27, 79 (1978) ; A. M. Ponte Goncalves, Chem. Phys. 19, 397 (1977).

[5] H. Morawitz, Phys. Rev. Letters 34, 1096 (1975).

[6] L. B. Coleman, M. J. Cohen, D. J. Sandman, F. G. Yamagishi, A. F. Garito, and A. J. Heeger, Solid State Commun. 12, 1125 (1973).

[7] J. D. Wright, K. Yakushi, and H. Kuroda, Acta Cryst. B 34, 1934 (1978).

[8] J. D. Wright, T. Ohta, and H. Kuroda, Bull. Chem. Soc. Japan 49, 2961 (1976).

[9] E. M. Engler, B. A. Scott, S. Etamad, T. Penney, and V. V. Patel, J. Amer. Chem. Soc. 99, 5909 (1977).

[10] H. Tsuchiya, F. Marumo, and Y. Saito, Acta Cryst. B 28, 1935 (1972).

\subsection{Phase transition and triplet exciton transport}

Whereas it was shown previously that the diffusion of triplet excitons into the preferred direction is not affected by the phase transition [4], this work demonstrates that also the energy diffusion into the other directions is not affected. This surprising result, although not fully understood, may be explained by the fact that during the phase transition the relative orientation of neighbouring donors is changed only slightly and thus the exchange integral remains virtually unchanged.

\section{Acknowledgement}

This work was performed while the authors were members of the University of Ulm, Dept. Exp. Phys. III. We like to thank Prof. Sackmann and our former colleagues for their help and advice. The work was supported by the Deutsche Forschungsgemeinschaft (DFG Mo 283/6).

[11] E. Erdle and H. Möhwald, Chem. Phys. 36, 283 (1979).

[12] J. J. Stezowski, J. Chem. Phys. 83, 550 (1979).

[13] H. Möhwald and A. Thaer, Phys. Stat. Sol. (a) 50, 131 (1978).

[14] J. Rosenthal, L. Yarmus, N. F. Berk, and W. Bizzaro, Chem. Phys. Lett. 56, 214 (1978).

[15] E. Erdle, Thesis, University of Ulm (West Germany) 1979.

[16] D. Haarer, Thesis, University of Stuttgart (West Germany) 1969.

[17] E. Erdle and H. Möhwald, to be published in phys. stat. sol. (b)

[18] J. Jortner, S. A. Rice, J. L. Katz, and S. Choi, J. Chem. Phys. 42, 309 (1965).

[19] W. Steudle, J. U. von Schütz, and H. Möhwald, Chem. Phys. Lett. 54, 461 (1978).

[20] H. Möhwald and E. Erdle, to be published in Chem. Phys. Lett.

[21] H. M. Vyas and A. M. Ponte Concalves, Chem. Phys. Lett. 51, 556 (1977). 